\title{
Self-Consistent Simulation of the Electric Field in a Rarefied Hypersonic Shock Layer
}

\author{
Erin D. Farbar* and Iain D. Boyd ${ }^{\dagger}$ \\ Department of Aerospace Engineering, University of Michigan, Ann Arbor, MI 48109
}

\begin{abstract}
During the hypersonic reentry of a vehicle into the Earth's atmosphere from space, the flow field energy is sufficient to produce ionizing reactions. The weakly ionized plasma around the vehicle can interfere with communications, and in some cases can have an important effect on the rate of heat transfer to the capsule surface. Additionally, the presence of charged particles in the plasma creates an electric field. In the present study, the modeling of the self-induced electric field in a simplified one-dimensional shock layer model is considered using a self-consistent DSMC-PIC method. The flow field results are compared to results obtained using an approximate DSMC method commonly used to incorporate the effect of the self-induced electric field on the structure of a rarefied, hypersonic shock layer. The DSMC-PIC results predict a higher electron temperature in the freestream region, a lower degree of ionization in the shock layer, and a larger contribution of surface heating from charged particle impact.
\end{abstract}

\section{Nomenclature}

d diameter in $\mathrm{m}$

E electric field in $\mathrm{V} / \mathrm{m}$

$\mathrm{k} \quad$ Boltzmann constant

$\mathrm{m}$ particle mass in $\mathrm{kg}$

M Mach number

$\mathrm{n}$ number density in $\mathrm{m}^{-3}$

q elementary charge in $\mathrm{C}$

$\mathrm{T}$ temperature in $\mathrm{K}$

$\mathrm{u} \quad$ velocity in $\mathrm{m} / \mathrm{s}$

$\rho$ density in $\mathrm{kg} / \mathrm{m}^{3}$

$\Delta \mathrm{t}$ simulation time step in $\mathrm{s}$

$\Delta \mathrm{x}$ grid spacing in $\mathrm{m}$

$\varepsilon_{o} \quad$ permittivity of free space, $\mathrm{F} / \mathrm{m}$

$\lambda$ mean free path in $\mathrm{m}$

$\lambda_{D} \quad$ Debye length in $\mathrm{m}$

$\omega_{p} \quad$ plasma frequency in $\mathrm{s}^{-1}$

$\phi \quad$ electric potential in $\mathrm{V}$

\section{Subscripts}

e electron value

i ion value

p particle value

$\mathrm{t}$ translational value

w wall value

*Graduate Student, Student Member AIAA. Email: efarbar@umich.edu

${ }^{\dagger}$ Professor, Associate Fellow AIAA. Email: iainboyd@umich.edu 


\section{Introduction}

The flow field about a vehicle undergoing a high-energy reentry into the Earth's atmosphere experiences collisions at energies sufficient to ionize particles. As a result, a weakly ionized plasma surrounds the vehicle and affects the flow field structure. A successful treatment of the effects of ionization on the flow field is important for predicting the condition of communications blackout, as well as for modeling the flow field radiation. This work deals specifically with the treatment of ionization effects during the rarefied, noncontinuum portion of the reentry trajectory.

Within a weakly ionized plasma there exists a self-induced ambipolar electric field, created by the large scale space-charge distribution of the plasma. This field is present to enforce the condition of charge neutrality in the bulk plasma, and within the bulk plasma the effect of this field is to cause the ions and electrons to diffuse at the same average rate. In the context of the geometry of the flow field around a typical reentry vehicle, the ambipolar field does not exist very near to solid boundaries, nor near the interface of the weak plasma and the neutral gas. In the sheath at the vehicle surface, there exists a large charge separation to prevent the electrons from diffusing to the surface. At the interface between the plasma and the neutral gas, a large charge separation exists to prevent the diffusion of the electrons into the freestream. In these regions the plasma is not charge neutral and the ambipolar diffusion assumption cannot be used to calculate the electric field.

Some researchers have reported results obtained from models of the ambipolar electric field of a weakly ionized reentry plasma in the literature. ${ }^{1-4}$ The common assumption used in all of these models is that the average velocity of the ions and electrons is equal throughout the domain, in order to either calculate the electric field or model it's effect on the flow structure. In all but the simulations by Taylor et al., ${ }^{1}$ the electrons were tied in some manner to the ions and not allowed to move freely throughout the domain. In the method introduced by Boyd ${ }^{2}$ to model the electric field effects, the velocity components of the charged particles were not modified to include the acceleration due to the field. The result of the various simplifications made in these previous models is that they all fail to model the electric field generated in a hypersonic reentry flow field in it's entirety and in a self-consistent manner.

The goal of this work is to capture all of the physics associated with the self-induced electric field and to determine which simplifications are valid in these types of flows. This is accomplished using a hybrid Direct Simulation Monte Carlo (DSMC) $)^{5}$ - Particle-in-Cell (PIC $)^{6}$ method. Results for a simplified one-dimensional model of the stagnation streamline of a rarefied hypersonic shock layer are presented in this paper. The lessons learned from this work will be used to model the shock layer around the FIRE II Earth reentry vehicle $^{7}$ at the $1631 \mathrm{~s}$ flight condition using the DSMC-PIC method.

\section{Details of the numerical method}

The computations presented in this work are performed using a DSMC code and a hybrid DSMC-PIC code which consists of the DSMC module coupled tightly to a PIC module. Each module is discussed separately below.

\section{II.A. DSMC module}

The DSMC module uses a DSMC code called MONACO, developed specifically for hypersonic flow simulations. Models are implemented in the code for rotational ${ }^{8}$ and vibrational energy exchange,${ }^{9}$ and chemical reactions in this study are simulated using the Total Collision Energy model. ${ }^{10}$ The current computations are performed in one dimension using the method due to Bird $^{11}$ for removing simulator particles in a onedimensional DSMC simulation of the flow along the stagnation streamline of a blunt body reentering an atmosphere.

When computing a flow field solution using only the DSMC module, quasi-neutrality of the plasma is enforced by invoking the assumption of ambipolar diffusion everywhere in the simulation domain. Because electrons are much lighter than ions and neutral particles, they possess a higher diffusion velocity. In order to retard the motion of the electrons, a space charge distribution is set up in the plasma that creates an 
electric field. As a result, the electrons are constrained to move at the same average velocity as the ions in the bulk plasma region. This assumption of ambipolar diffusion of the charged species is used in the DSMC code to maintain quasi-neutrality in the plasma without explicitly computing the plasma potential and electric fields. In the implementation used here, the average ion velocity is computed in each cell, and the electrons are moved with this average velocity while retaining their large thermal velocity components. ${ }^{2}$ The collision algorithm is subcycled so that the high collision rate of the electrons with heavy particles is accurately represented. Further details of this model are found in Ref. 12.

\section{II.B. PIC module}

The PIC module uses the charge-in-cloud (CIC) interpolation method ${ }^{6}$ to resolve the space-charge density at the nodes of the computational grid using the electron and ion simulator particles in each cell. The space charge density is then used as the source term in the solution of the electrostatic potential equation given by Equation 1. The electric field is computed from the potential using Equation 2 and interpolated from grid nodes back to the charged particles again using the CIC method. Assuming that the instantaneous electric field is constant during a simulation time step, the average velocity of a charged particle during one iteration of the simulation is computed using Equation 3. This is the velocity that charged particles are moved with during the move portion of the DSMC algorithm. The velocity increment due to the field acceleration given by Equation 4 is added to the axial velocity components of the charged particles. This routine is completed at every DSMC iteration following the move portion of the DSMC algorithm, making the DSMC-PIC code tightly coupled.

$$
\begin{gathered}
\frac{d^{2} \phi}{d z^{2}}=-\frac{q}{\varepsilon_{o}}\left(n_{i}-n_{e}\right) \\
E_{z}=-\frac{d \phi}{d z} \\
\overline{u_{p}^{\prime}}=u_{p}+\frac{1}{2} \Delta u \\
\Delta u=\frac{q}{m} E_{z} \Delta t
\end{gathered}
$$

\section{Details of the simplified shock layer model}

The simplified shock layer model is a one-dimensional model of the stagnation streamline of an axisymmetric, hypersonic shock layer that is formed during the reentry of a blunt body into the atmosphere. The purpose of the simplified model is to provide a test case that allows the structure of a hypersonic shock layer to be simulated using the DSMC-PIC method, but at a lower computational cost than would be required to simulate a shock layer formed at Earth reentry conditions. This allows the sensitivity of the solution to numerical parameters such as $\lambda_{D} / \Delta x$ and $\omega_{p} \times \Delta t$ to be addressed in a timely manner, while still providing valuable insight into the fundamental physics associated with this type of flow field.

The freestream of the simplified shock layer model is composed entirely of very low density atomic nitrogen. The diameter of the nitrogen particles is increased by three orders of magnitude in order to yield a shock stand-off distance and mean free path typical of a reentry vehicle in the rarefied portion of the atmosphere. A single ionizing reaction is defined to create plasma in the shock layer, as given by Equation 5, and the rate given in the literature ${ }^{13}$ is increased by three orders of magnitude to yield a degree of ionization comparable to the FIRE II flight condition. Additionally, the electron mass is increased by three orders of magnitude to yield $\mathrm{m}_{i} / \mathrm{m}_{e}=25$. The wall temperature is set to $460 \mathrm{~K}$ and the wall is diffuse and fully catalytic to the recombination of charged species but not to atoms.

$$
N+N \rightarrow N^{+}+N+e, \quad \mathrm{k}=2 \times 10^{-10} \mathrm{e}^{-168200 / T} \mathrm{~m}^{3} / \mathrm{s}
$$

The potential boundary condition at the freestream is of von Neumann type, and set to $\mathrm{E}_{z}=\mathrm{d} \phi / \mathrm{dz}=$ $0 \mathrm{~V} / \mathrm{m}$ in order to enforce a zero electric field condition in the far-field. The potential boundary condition on the vehicle surface is fixed at $\phi=0 \mathrm{~V}$, and current is permitted to flow to the surface. A summary of 
the model parameters for the simplified shock layer model is given in Table 1. A schematic of the simplified shock layer model is given in Figure 1.

The numerical mesh consists of 1000 uniform grid points. This yields a ratio $\lambda_{D} / \Delta \mathrm{x}=5$, evaluated at the location of peak ionization in the shock layer. The timestep used is dictated by the minimum cell crossing time of the electron particles, and is set to $1 \times 10^{-9} \mathrm{~s}$, which is much less than the plasma period. The particle weight is selected to yield approximately 40 charged particles per cell in the peak plasma density region. A total of 250000 particles are simulated. Convergence of the simulation is defined to have been reached when both the number of particles in the domain and the current to the probe surface have reached steady state. This typically occurs in approximately 5 ion transit times defined by $\mathrm{T}_{\text {transit }}=\mathrm{L} / \mathrm{V}_{t h, i}$, where $\mathrm{L}$ is the shock layer thickness and $\mathrm{V}_{t h, i}$ is the ion thermal velocity based on the peak shock temperature. After a steady state has been reached, a minimum of 40000 sampling iterations are performed. For some quantities such as the electron velocity distribution function, many more samples are collected in order to obtain statistically meaningful results. The total simulation time is approximately ten hours on a single processor. The numerical parameters used in the simulation are summarized in Table 2.

\section{Results and Discussion}

\section{IV.A. Results obtained using the DSMC code with the ambipolar assumption}

The simplified shock layer model is first computed using the DSMC algorithm and the assumption of ambipolar diffusion in order to move the electrons through the grid.

A general impression of the flow field is given in Figures 2 and 3. Figure 2 shows the number densities of the species present in the simulation as a function of distance from the stagnation point at $\mathrm{x}=0 \mathrm{~m}$. The shock begins to form at approximately $-0.07 \mathrm{~m}$. The flow is weakly ionized, with a maximum degree of ionization of approximately $8 \%$. Figure 3 shows the total translational and electron temperatures along the stagnation streamline. A peak temperature of approximately $41000 \mathrm{~K}$ is reached in the simulation, and the electrons are very nearly in thermal equilibrium with the bulk gas in the shock layer. While the electrons and ions are born with low energies in the ionizing reaction, they gain energy upon collisions with high energy $\mathrm{N}$ atoms from the incoming flow. Because the mass of the electrons has been artificially increased, their energy transfer efficiency in elastic collisions is nearly that of the ions, and as such they become nearly thermally equilibrated with the bulk gas. In the region upstream of the shock layer, the electrons remain at a higher temperature than the bulk flow. This is a result of artificially increasing the electron mass which in turn reduces their collision rate, and has been observed in other reentry simulations performed using the DSMC method that utilized heavier electrons. ${ }^{14}$

\section{IV.B. Results obtained using the DSMC-PIC model}

The simplified shock layer model is computed using the DSMC-PIC code, in which the electrons are no longer constrained by the average ion velocity, and instead allowed to move freely about the domain. The macroscopic flow field results obtained by sampling the flow field solution once a steady state has been reached are presented in the following figures.

Figure 4 shows the surface charge accumulated on the vehicle during the simulation. After the initial transient, the surface charge accumulation exhibits a positive and linear slope, indicating that a steady state has been reached with a constant, positive net current flowing to the surface. The magnitude of the current is on the order of $\mu A$, which indicates that the voltage predicted at the vehicle surface given by this model, relative to a reference in the freestream, is very close to what would be predicted by treating the vehicle as a floating surface. If the vehicle were modeled as an electrically floating surface, the voltage at the vehicle surface would adjust itself until the net current to the vehicle was zero.

Figure 5 shows the electrostatic potential and electric field throughout the simulation domain. In the shock layer there is a negative electric field that exists to inhibit the diffusion of the electrons away from the shock layer. At the vehicle surface, there is a very thin region of a strong, positive electric field. This is the wall sheath region, and the field exists here to inhibit the diffusion of the electrons to the vehicle surface. The negative electric field reaches a minimum of approximately $200 \mathrm{~V} / \mathrm{m}$, while the field in the sheath region reaches a maximum of approximately $600 \mathrm{~V} / \mathrm{m}$. While the definition of the beginning of the wall sheath is somewhat arbitrary, it is clear that the potential drop in the sheath region is approximately $1 \mathrm{~V}$. A value of $2 \mathrm{~V}$ is predicted by collisionless sheath theory, using Equation 6 and the plasma parameters at the edge of 
the sheath. The difference between the two values can be attributed to the fact that the mean free path is almost an order of magnitude smaller than the Debye length in the near wall region.

$$
\phi_{w}=-\frac{k T_{e}}{2 q} \ln \left(\frac{T_{e}}{T_{i}} \frac{m_{i}}{m_{e}}\right)
$$

Figure 6 shows the profiles of the electron and ion number density through the shock layer, as well as the magnitude of the charge separation. The separation of the ions and electrons upstream of the shock layer occurs because the densities of charged particles, and therefore the attainable charge separation, are too low in that region to produce a sufficient electric field to retard the diffusion of the electrons. Thus, the diffusion mechanism transitions from ambipolar to free diffusion in the region upstream of the shock layer. In the sheath region at the vehicle surface, a very large charge separation exists to produce the strong electric fields necessary to slow the diffusion of the electrons to the vehicle surface. Figure 7 shows a magnified view of the sheath region of the flow. Given that the Debye length in this region of the flow is approximately $0.001 \mathrm{~m}$, the sheath is between 5-10 Debye lengths in width in this flow as expected.

Figure 8 shows the velocity increment imposed on both the electrons and the ions by the electric field at each time step throughout the flow. The ions see a minimum of $-1.5 \mathrm{~m} / \mathrm{s}$ and a maximum of $4 \mathrm{~m} / \mathrm{s}$ per timestep, while the electrons see a minimum of $-100 \mathrm{~m} / \mathrm{s}$ and a maximum of $35 \mathrm{~m} / \mathrm{s}$ per time step.

In order to assess the effect of modeling the self-induced electric field in a consistent way on the flow field properties of interest, it is instructive to compare the flow field properties obtained using both the DSMC and DSMC-PIC methods. Figure 9 shows the profiles of velocity along the stagnation streamline obtained using both methods. It is clear that there is little change in this flow field property by moving to the higher fidelity DSMC-PIC method, and an examination of the translational temperature and density profiles along the stagnation streamline yields the same conclusion.

Figure 10 shows a comparison of the number densities of electrons and ions along the stagnation streamline obtained with the DSMC and DSMC-PIC methods. Because the electrons are not artificially constrained to move with the ions in the DSMC-PIC model, the electrons diffuse further towards the freestream as indicated by their higher number density there than that predicted by the DSMC model. Due to the selfinduced electric field, the ions also diffuse further into the freestream region. As a result, the flow field solution obtained with the DSMC-PIC model predicts lower charged species mole fractions in the near vehicle region, and higher charged species mole fractions in the freestream region than are predicted by the DSMC model alone. The peak electron number density predicted by the DSMC-PIC model is approximately $60 \%$ of that predicted by the DSMC model.

Figure 11 shows a comparison of the average velocities of each species along the stagnation streamline. In the freestream region, the use of the DSMC-PIC model results in a decrease in the average ion velocity and a large decrease in the average electron velocity. The decrease in average ion velocity is a result of their deceleration caused by the negative electric field in the freestream region. The decrease in the average electron velocity is likely a combined effect. Because the electrons are uncoupled from the ions in the DSMCPIC simulation, they are free to move away from the vehicle surface, meaning that electrons at the negative tail of the distribution function will populate the freestream region. Additionally, the particles that arrive at the freestream region where the electric field is nearly zero are those with large enough negative velocities to overcome the deceleration felt at the peak of the negative portion of the electric field at $\mathrm{x}=-0.05 \mathrm{~m}$. This phenomenon will also result in a negative average electron velocity in the freestream region. In the sheath region, the average ion velocity obtained from the DSMC-PIC simulation shows a slight increase over that from the DSMC simulation, as the ions are accelerated through the potential drop in the sheath. The average electron velocity shows a larger increase, an effect again attributable to the fact that only the very high energy electrons can traverse the potential drop in the sheath, resulting in a higher average velocity in the sheath region than in the pre-sheath region of the flow.

Figure 12 shows a comparison of the translational and electron translational temperatures obtained using the DSMC and DSMC-PIC methods. While there are only small changes in the sheath region of the flow field, there is a marked difference between the two predictions over a portion of the shock layer and in the freestream region. While the DSMC simulation predicts near thermal equilibrium between the electron and translational temperatures, the electrons are very clearly not in thermal equilibrium in the results obtained using the DSMC-PIC method, and the electron translational temperature is very high. This occurs because when the electrons are allowed to move freely, there are some very fast electrons that escape toward the edge of the domain and freely diffuse. That is, there are some electrons that have left the shock layer with large 
negative velocities and experience few collisions in the low density ambient region of the simulation, but there are some electrons which readily collide and equilibrate in the high density shock layer. This results in a distribution function of electron velocities that is broadened and somewhat double-peaked in nature. To illustrate this point, Figure 13 shows the normalized electron velocity distribution at the $\mathrm{x}=-0.1 \mathrm{~m}$ location in the domain obtained using the DSMC and DSMC-PIC methods. This location is just upstream of the shock layer. It is clear that the distribution function obtained from the DSMC solution is much more narrow than that obtained from the DSMC-PIC solution, and is centered about the freestream velocity. Thus, the DSMC method predicts near thermal equilibrium between the electrons and bulk flow at $\mathrm{x}=-0.1 \mathrm{~m}$, while the DSMC-PIC solution does not. Additionally, Figure 14 shows the normalized electron velocity distribution obtained from the DSMC-PIC solution at the $\mathrm{x}=-0.15 \mathrm{~m}$ location in the domain, which is further upstream of the shock. Also shown on this figure is the Gaussian distribution corresponding to the standard deviation and mean calculated from the sampled electron velocities. The distribution appears to be double peaked, however the very small number of electron simulator particles in the freestream region of the domain make it difficult to extract a more conclusive distribution function in that region. Fundamentally it is clear, however, that the distribution function is wide and not Maxwellian, which results in the large electron temperatures predicted by the DSMC-PIC method in the region upstream of the shock shown.

Table 3 shows a comparison of various heat flux quantities obtained from the DSMC and DSMC-PIC solutions. The contribution to the surface heat flux due to charged particle impact predicted by the DSMCPIC model is larger than that predicted by the DSMC model by $22 \%$. This difference yields a $1 \%$ increase in the predicted contribution of heating due to charged particle impact to the total vehicle heat flux for these freestream conditions. The increase in the heat flux from charged particle impact is largely due to the acceleration of the heavier ions in the sheath near the vehicle surface.

\section{Conclusions}

The flow field and electric field predictions obtained with the DSMC-PIC method for the simplified shock layer model are consistent both quantitatively and qualitatively with electrostatic theory. Specifically, the potential drop of approximately $1 \mathrm{~V}$ predicted in the sheath region at the probe surface is close to that predicted by collisionless sheath theory, differing due to the collisionality of the sheath plasma. The transition to free diffusion from ambipolar diffusion upstream of the shock layer is apparent in the flow field solution as expected. The predicted sheath width is on the order of 5-10 Debye lengths, as expected.

Comparison of the DSMC results obtained by employing the ambipolar diffusion assumption to the DSMC-PIC results indicate that the use of the ambipolar assumption has no discernable effect on the prediction of the bulk velocity, translational temperature, or density through the shock layer for these freestream parameters. However, the use of the self-consistent DSMC-PIC model results in a decrease in the predicted average ion and electron velocities in the shock layer, and an increase in the predicted average ion and electron velocities in the sheath region. In the shock layer, the electron number density predicted by the DSMC-PIC model is approximately $60 \%$ of that predicted by the DSMC model. The use of the self-consistent model results in a significant increase in the predicted electron translational temperature at the near-freestream edge of the shock layer and in the freestream. This temperature increase may have important implications for the prediction of ionization due to electron impact mechanisms. Lastly, the selfconsistent DSMC-PIC model predicts a $22 \%$ increase in the contribution of heat flux to the vehicle surface due to charged particle impact.

The knowledge gained from experimenting with the DSMC-PIC model of the simplified shock layer will be used to develop a model of the shock layer that was formed in front of the FIRE II Earth reentry probe at the $1631 \mathrm{~s}$ flight condition.

\section{Acknowledgments}

The authors gratefully acknowledge the financial support provided by NASA grant NCC3-989. E. Farbar also gratefully acknowledges the financial support of the National Research Council of Canada and the Zonta International Foundation. 


\section{References}

${ }^{1}$ Taylor, J. C., Carlson, A. B., and Hassan, H. A., "Monte Carlo Simulation of Radiating Re-entry Flows," Journal of Thermophysics and Heat Transfer, Vol. 8, No. 3, 1994, pp. 478-485.

${ }^{2}$ Boyd, I. D., Trumble, K., and Wright, M. J., "Nonequilibrium Particle and Continuum Analyses of Stardust Entry for Near-Continuum Conditions," 38th AIAA Aerospace Sciences Meeting and Exhibit, 2007, AIAA-2007-4543.

${ }^{3}$ Bird, G. A., "Nonequilibrium Radiation During Re-entry at $10 \mathrm{~km} / \mathrm{s} . "$ AIAA 22nd Thermophysics Conference, 1987, AIAA-87-1543

${ }^{4}$ Gallis, M. A. and Harvey, J. K., "Ionization Reactions and Electric Fields in Plane Hypersonic Shock Waves," Rarefied Gas Dynamics, Vol. 160 of Progress in Astronautics and Aeronautics, AIAA, New York, 1992, pp. 234-244.

${ }^{5}$ Bird, G. A., Molecular Gas Dynamics and the Direct Simulation of Gas Flows, Oxford Science Publications, 1994.

${ }^{6}$ Hockney, R. W. and Eastwood, J. W., Computer Simulation Using Particles, McGraw-Hill Inc., 1981.

${ }^{7}$ Cornette, E. S., "Forebody Temperatures and Calorimeter Heating Rates Measured During Project Fire II Reentry at 11.35 Kilometers Per Second," Tech. Rep. NASA TM X-1305, Langley Research Center, 1966.

${ }^{8}$ Boyd, I. D., "Rotational-translational Energy Transfer in Rarefied Nonequilibrium Flows," Physics of Fluids, Vol. 2, No. 3, 1989, pp. 447-452.

${ }^{9}$ Boyd, I. D., "Analysis of Vibrational-translational Energy Transfer Using the Direct Simulation Monte Carlo Method," Physics of Fluids, Vol. 3, No. 7, 1991, pp. 1785-1791.

${ }^{10}$ Bird, G. A., "Monte Carlo Simulation in an Engineering Context," Rarefied Gas Dynamics, edited by S. S. Fisher, Vol. 74 of Progress in Astronautics and Aeronautics, AIAA, New York, 1981, pp. 239-255.

${ }^{11}$ Bird, G. A., "Direct Simulation of Typical AOTV Entry Flows," AIAA/ASME 4th Joint Thermophysics and Heat Transfer Conference, 1986, AIAA-86-1310.

${ }^{12}$ Farbar, E. D. and Boyd, I. D., "Simulation of Fire II Reentry Flow Using the Direct Simulation Monte Carlo Method," 40th AIAA Thermophysics Conference, 2008, AIAA 2008-4103.

${ }^{13}$ Carlson, L. A. and Gally, T. A., "The Effect of Electron Temperature and Impact Ionization on Martian Return AOTV Flowfields," 24th AIAA Thermophysics Conference, 1989, AIAA-89-1729.

${ }^{14}$ Boyd, I. D., "Modeling of associative ionization reactions in hypersonic rarefied flows," Physics of Fluids, Vol. 19, 2007, Article 096102. 
Table 1: Model parameters for the simplified shock layer model.

\begin{tabular}{lc}
\hline \hline Property & Value \\
\hline$n_{\infty}$ & $2 \times 10^{14} \mathrm{~m}^{-3}$ \\
$\mathrm{U}_{\infty}$ & $11.37 \mathrm{~km} / \mathrm{s}$ \\
$\mathrm{T}_{\infty}$ & $212 \mathrm{~K}$ \\
$\mathrm{~T}_{w}$ & $460 \mathrm{~K}$ \\
$\mathrm{M}_{\infty}$ & 25 \\
$\lambda_{\infty}$ & $4.5 \times 10^{-3} \mathrm{~m}$ \\
$\lambda_{D}$ & $1.0 \times 10^{-3} \mathrm{~m}$ \\
\hline \hline
\end{tabular}

Table 2: Numerical parameters for the simplified shock layer model.

\begin{tabular}{lc}
\hline \hline Property & Value \\
\hline $\mathrm{Wp}$ & $5 \times 10^{6}$ \\
$\omega_{p} \times \Delta \mathrm{t}$ & 0.03 \\
$\lambda_{D} / \Delta \mathrm{x}$ & 5 \\
\hline \hline
\end{tabular}

Table 3: Comparison of surface heat flux predicted by the DSMC and DSMC-PIC models.

\begin{tabular}{lcc}
\hline \hline Model & charged particle heat flux $\left(\mathrm{W} / \mathrm{m}^{2}\right)$ & $\%$ total heat flux due to charge particle impact \\
\hline DSMC & $7.7 \times 10^{-2}$ & $17 \%$ \\
DSMC-PIC & $9.4 \times 10^{-2}$ & $18 \%$ \\
\hline \hline
\end{tabular}




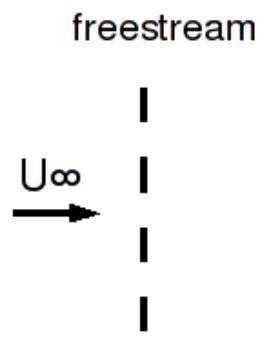

$E z=0$

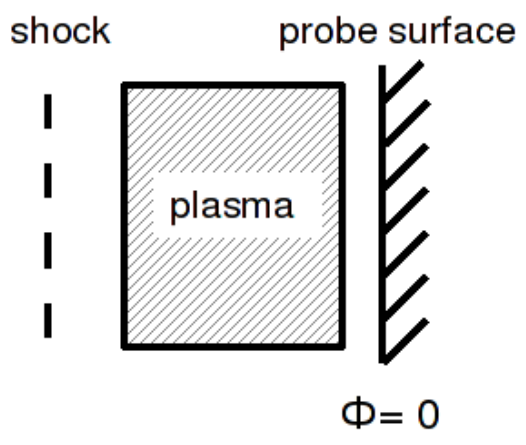

Figure 1: Schematic of the simplified shock layer model.

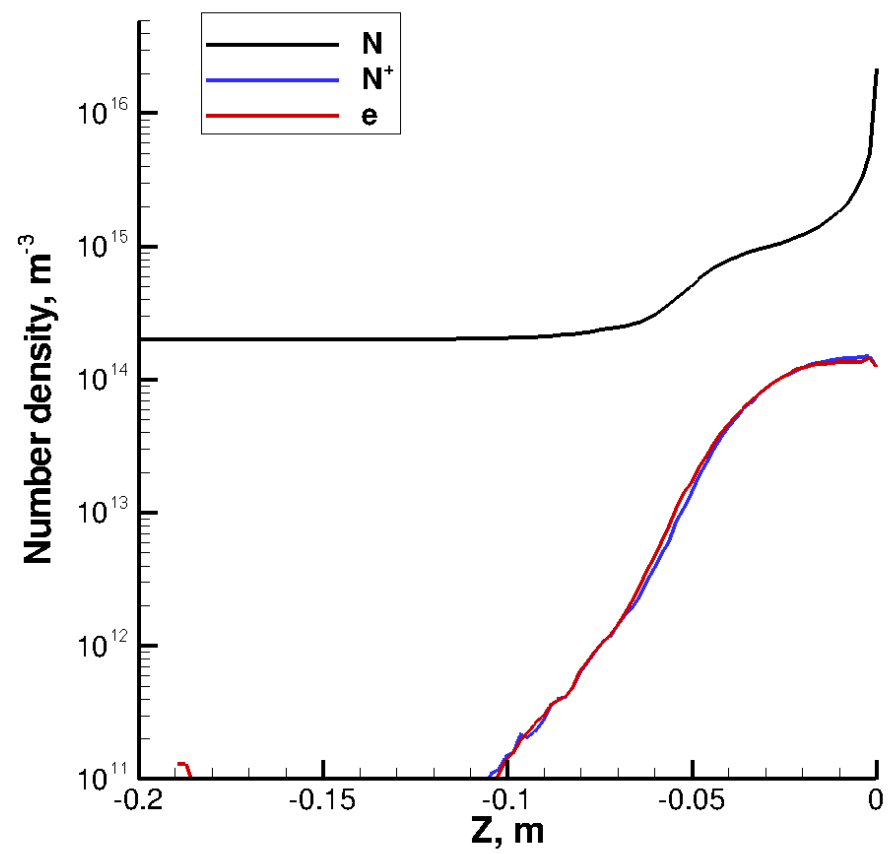

Figure 2: Number densities along the stagnation streamline from the DSMC solution. 


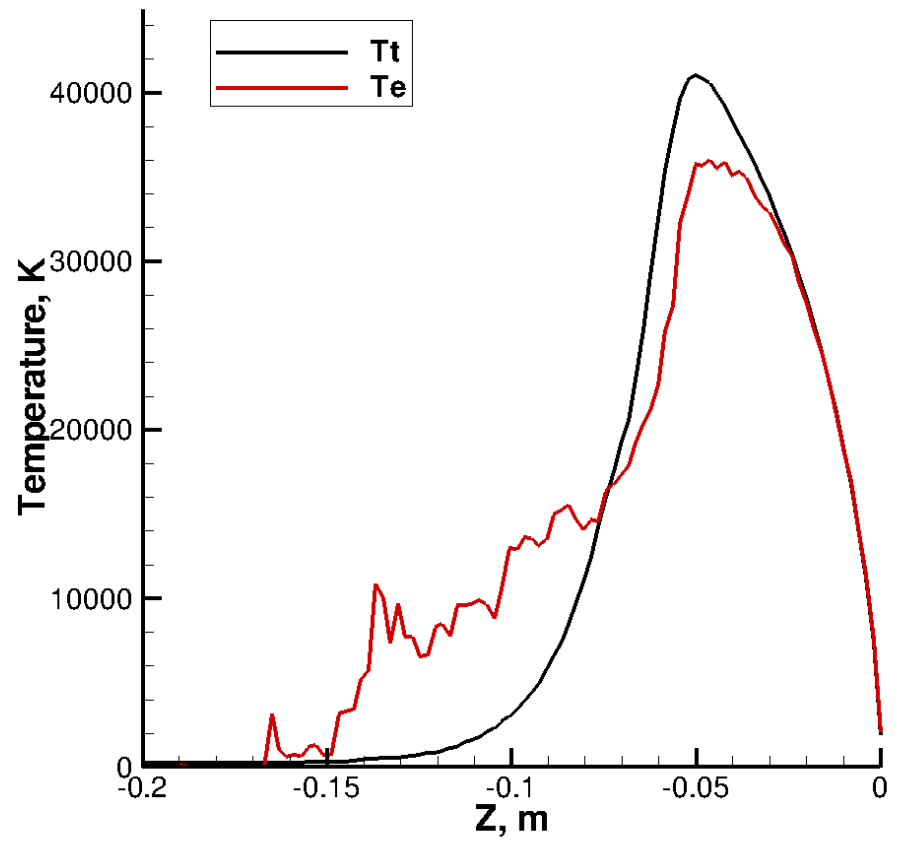

Figure 3: Translational temperatures along the stagnation streamline from the DSMC solution.

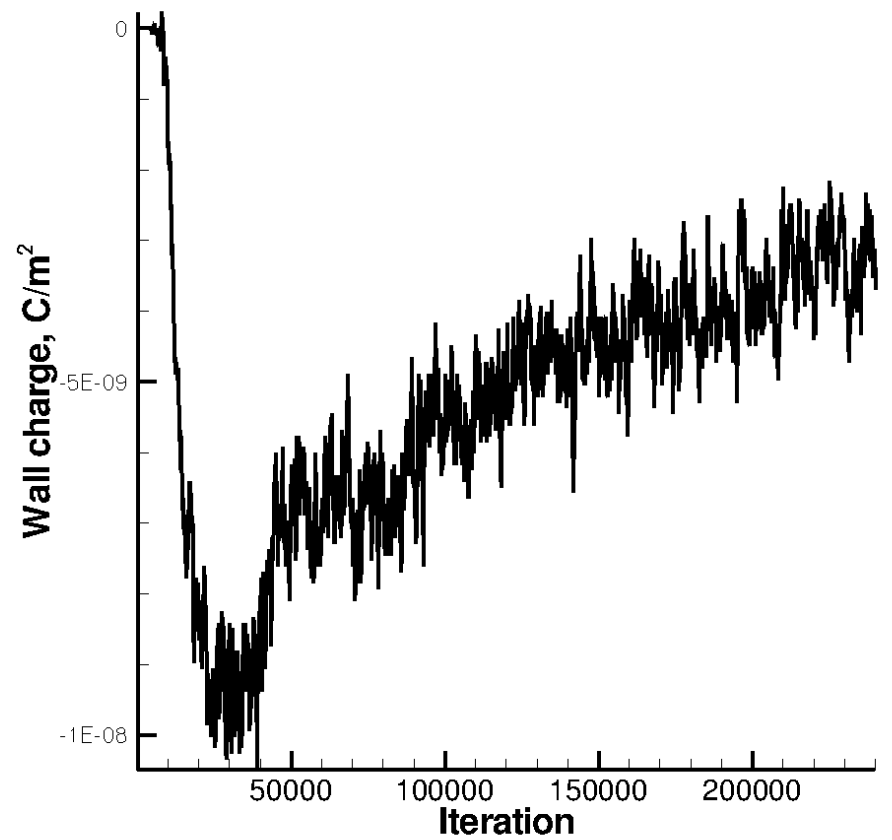

Figure 4: Charge on vehicle surface during DSMC-PIC simulation. 


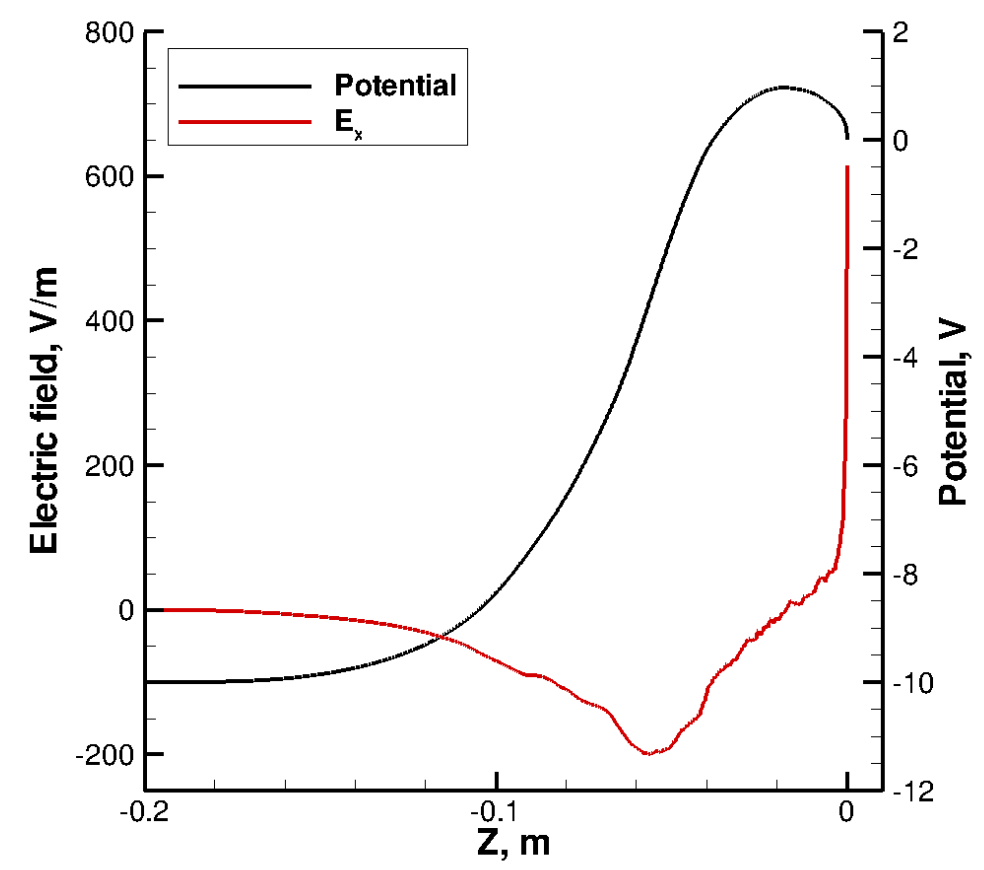

Figure 5: Electric potential and electric field along the stagnation streamline.

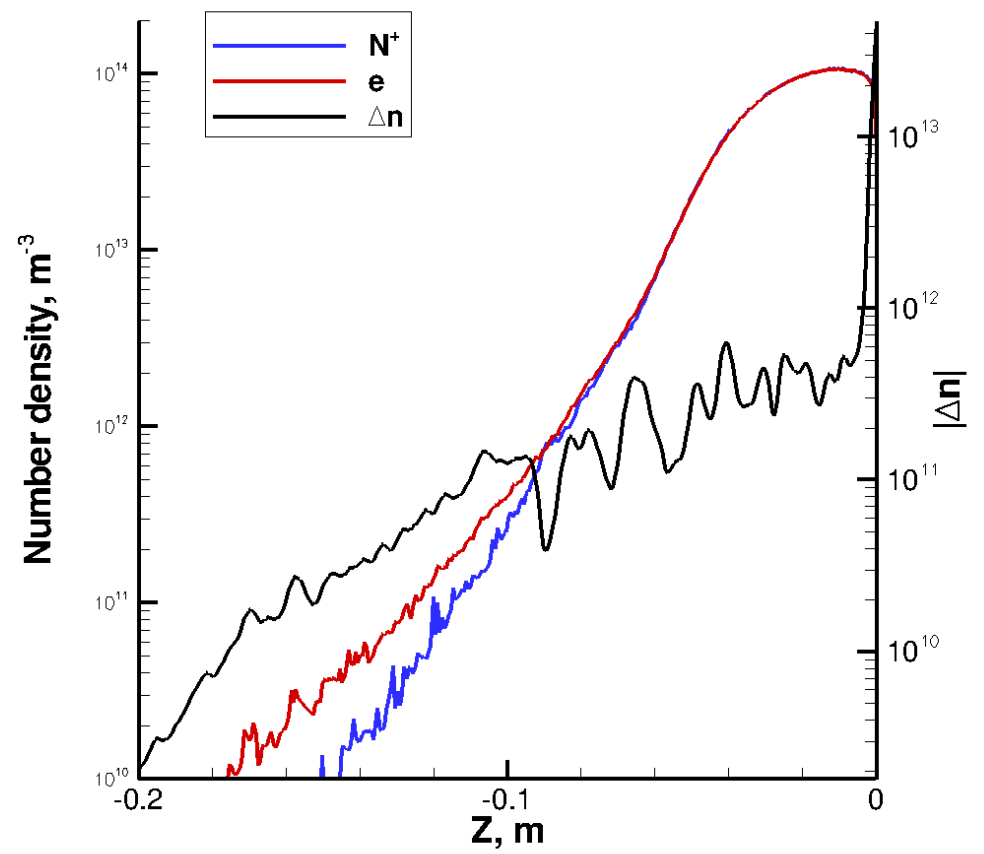

Figure 6: Number densities of charged species and charge separation along the stagnation streamline from the DSMC-PIC solution. 


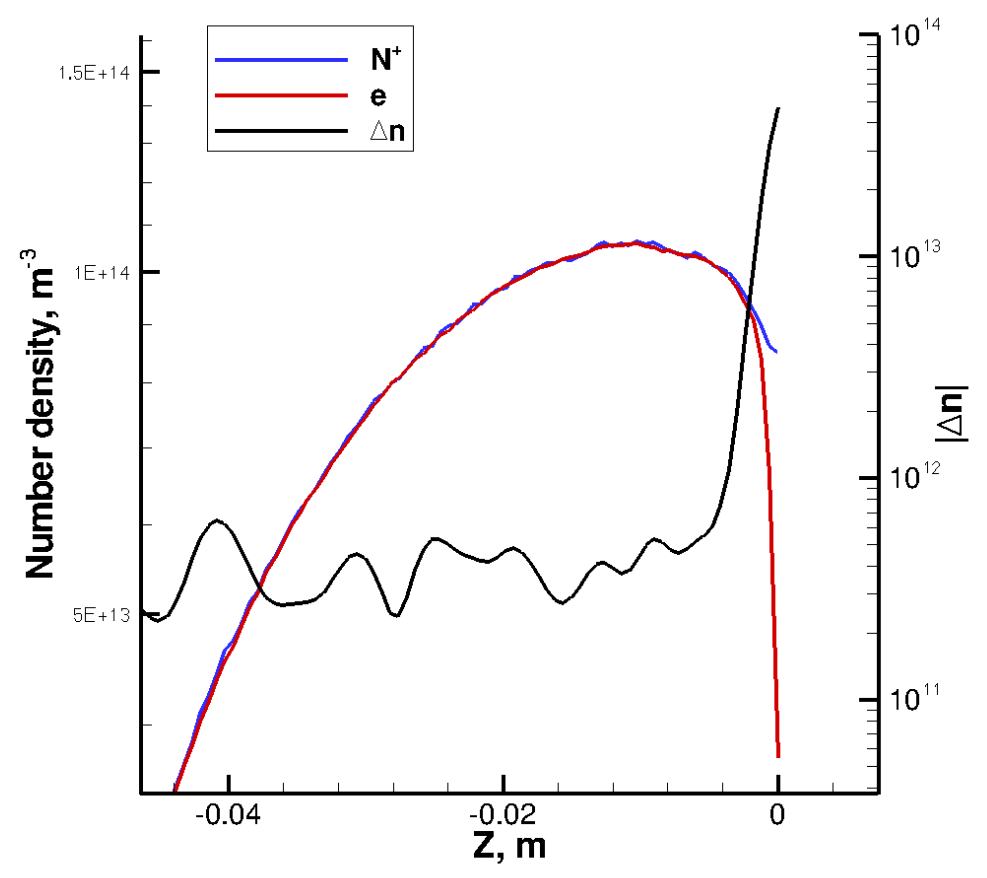

Figure 7: Number densities of charged species and charge separation along the stagnation streamline from the DSMC-PIC solution.

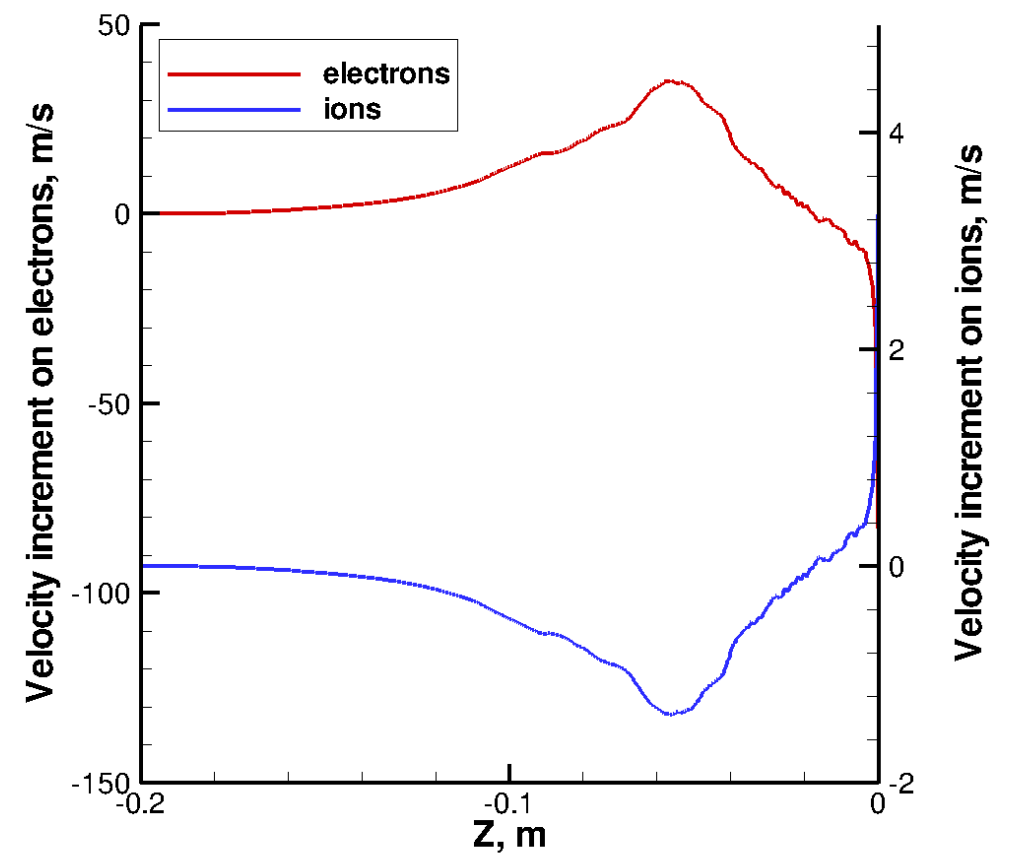

Figure 8: Velocity increments on charged particles due to the electric field. 


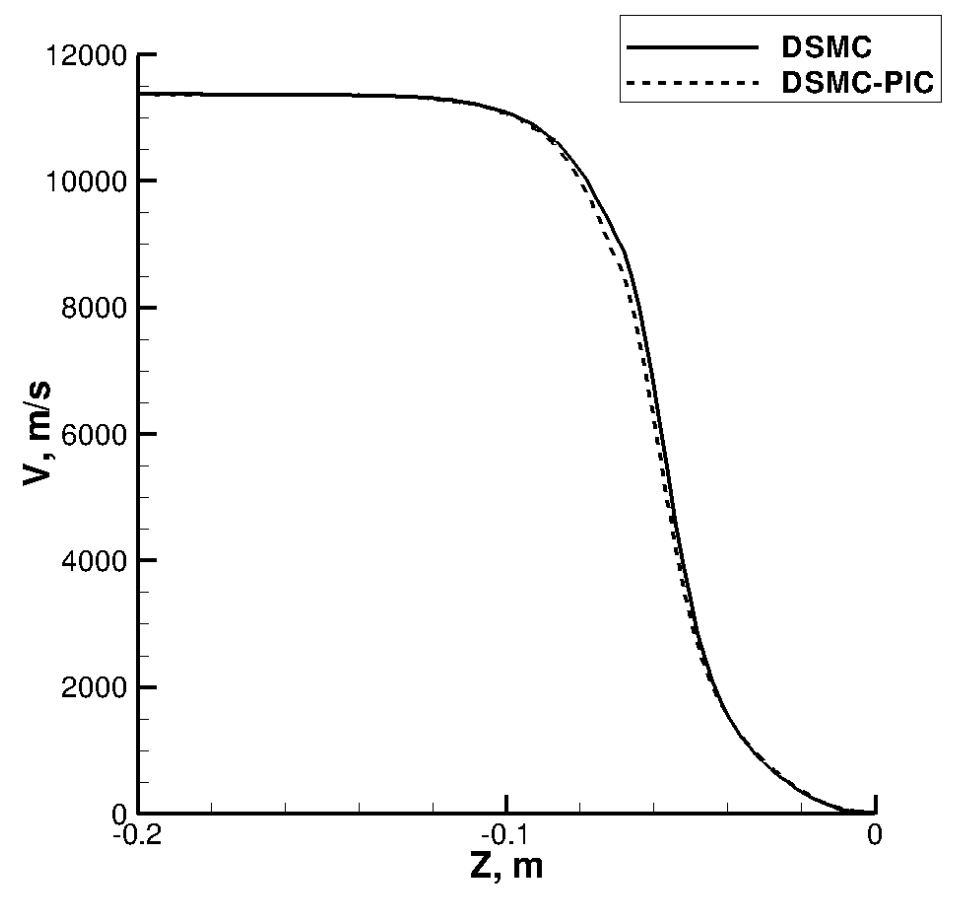

Figure 9: Velocity along the stagnation streamline obtained using the DSMC and DSMC-PIC methods.

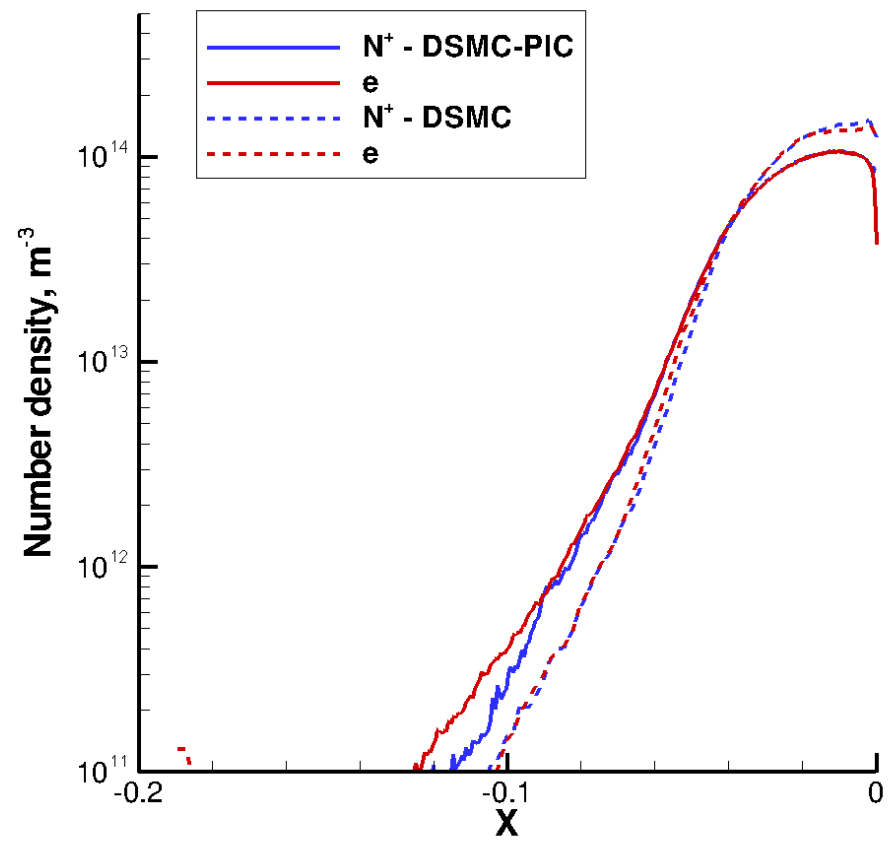

Figure 10: Number densities of individual species along the stagnation streamline obtained using the DSMC and DSMC-PIC methods. 


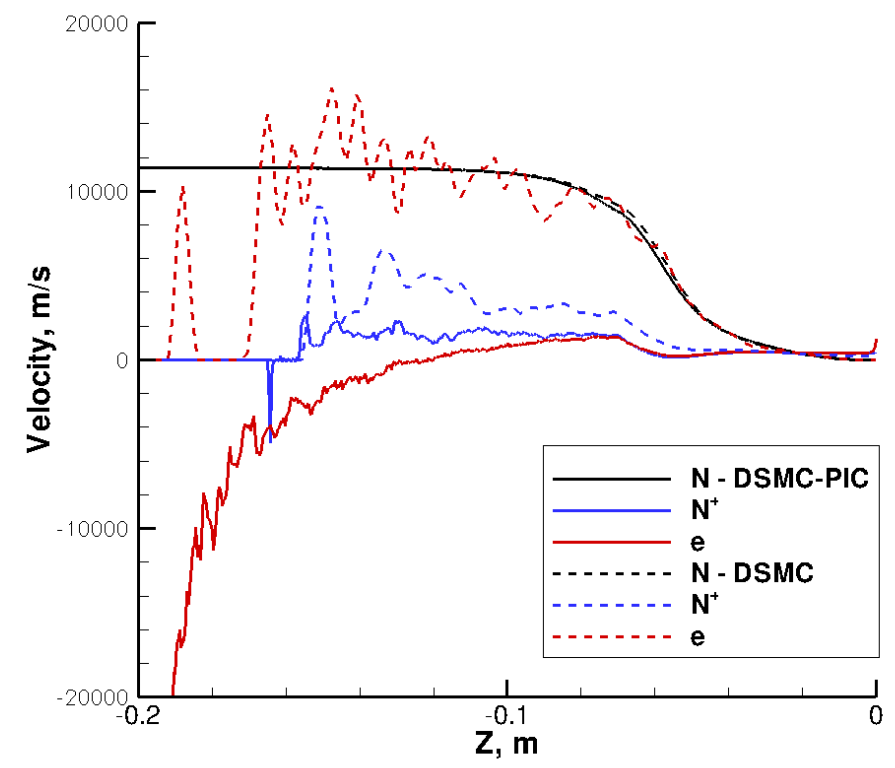

Figure 11: Average velocities of individual species along the stagnation streamline obtained using the DSMC and DSMC-PIC methods.

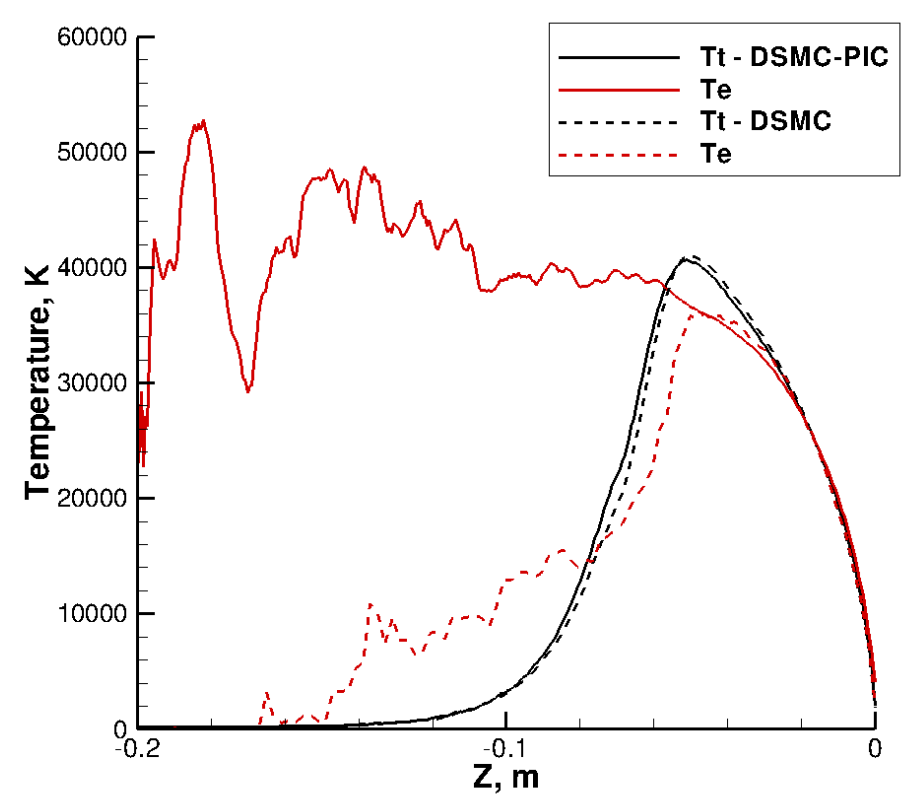

Figure 12: Translational and electron temperatures along the stagnation streamline obtained using the DSMC and DSMC-PIC methods. 


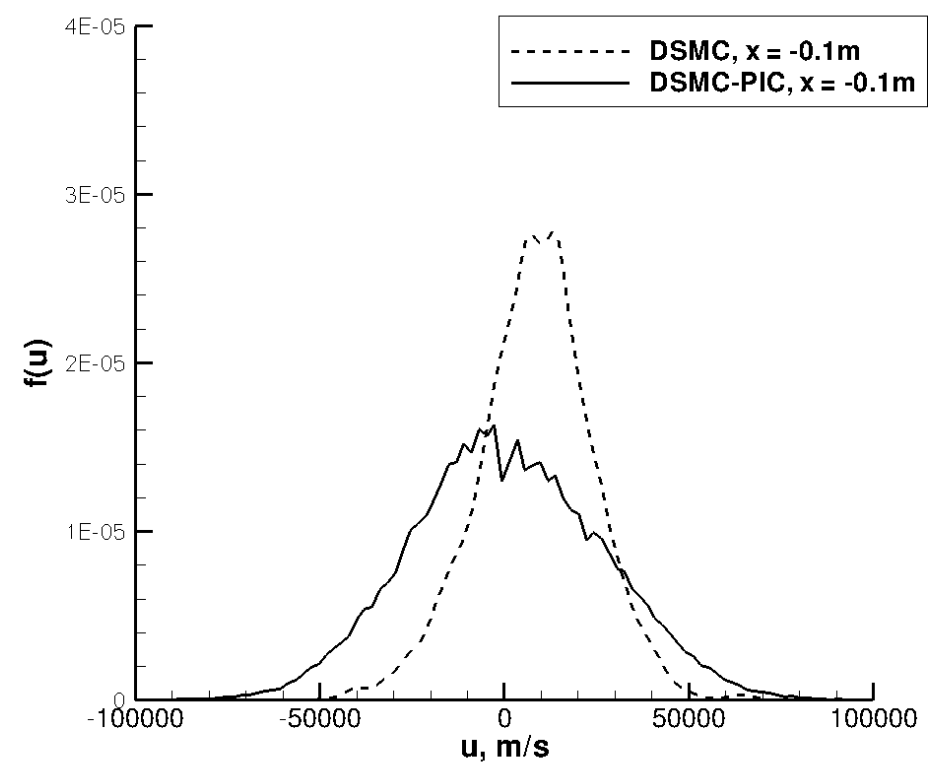

Figure 13: Electron velocity distribution obtained from the DSMC and DSMC-PIC solutions at $\mathrm{x}=-0.1 \mathrm{~m}$.

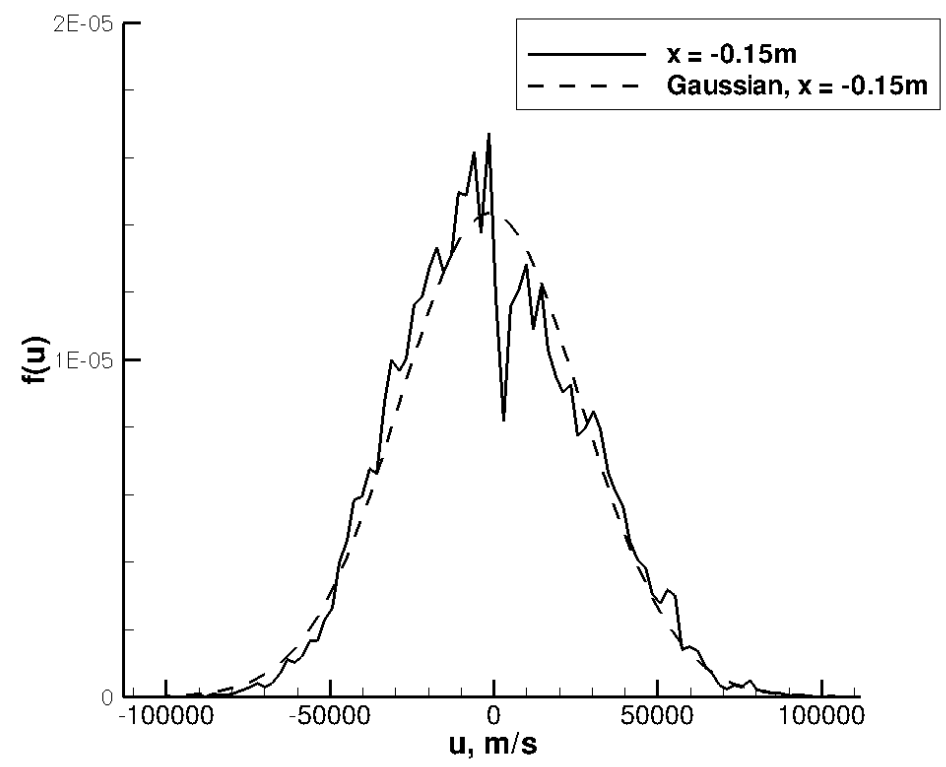

Figure 14: Electron velocity distribution obtained from the DSMC-PIC solution at $\mathrm{x}=-0.15 \mathrm{~m}$. 